\title{
Breastfeeding promotion: evidence and problems
}

\author{
Roberto Bellù, Manuela Condò \\ Neonatal Intensive Care Unit, Manzoni Hospital, Lecco, Italy
}

\begin{abstract}
Although breastfeeding is associated with many health benefits in children and mothers, and World Health Organization (WHO) recommends exclusive breastfeeding until 6 months of age and continued breastfeeding until 2 years of age, overall breastfeeding rates remain low. Italian rates of exclusive breastfeeding do not differ from international data. The aim of this review is to evaluate evidence of breastfeeding promotion interventions and the remaining problems to achieve them. We found that breastfeeding support is a complex system of interventions, including individual, structural and environmental factors. Many systematic reviews report evidence that breastfeeding support offered to women increases duration and exclusivity of breastfeeding, both in full term healthy newborns and in preterm infants. Political and economic efforts should be made to ensure breastfeeding support to all women in the different settings, assuming it as a collective target.
\end{abstract}

\section{Introduction}

The World Health Organization (WHO) recommendations are feeding infants exclusively on breast milk until 6 months of age

Correspondence: Roberto Bellù, Neonatal Intensive Care Unit, Manzoni Hospital, via Eremo 9, 23900 Lecco, Italy.

Tel. +39.0341.489231- Fax +39.0341.489247.

E-mail: r.bellu@asst-lecco.it

Key words: Breastfeeding; Newborns; NICU.

Contributions: the authors contributed equally.

Conflict of interest: the authors declare no potential conflict of interest.

Funding: Funded by Merqurio Editore S.r.l., with the unconditional contribution of Nestlè Italiana S.p.A.

Received for publication: 7 June 2017.

Accepted for publication: 20 June 2017.

This work is licensed under a Creative Commons Attribution

NonCommercial 4.0 License (CC BY-NC 4.0).

CCopyright R. Bellù and M. Condò, 2017

Licensee PAGEPress, Italy

La Pediatria Medica e Chirurgica 2017; 39:156

doi:10.4081/pmc.2017.156 and continuing to breastfeed as an important part of the infant's diet until 2 years of age, ${ }^{1}$ but overall breastfeeding rates are still below international goals. ${ }^{2}$

Data for exclusive breastfeeding in Italy are reported in Table 1. It is clear that despite high rates of early exclusive breastfeeding, prolonged exclusive breastfeeding rates, as for WHO recommendations, are quite low.

Despite WHO defined in 2008 indicators to study feeding practices in children ${ }^{7}$ (early initiation of breastfeeding, exclusive breastfeeding before 6 months of life, continued breastfeeding at 1 and at 2 years of life), there are few reports from high-income countries to allow a good-quality comparison of data, as recently reported by Victora. ${ }^{8}$ Authors found a strong inverse correlation between breastfeeding and gross domestic product per person and between unsatisfactory rates of early initiation and exclusive breastfeeding in all settings (high-income, middle-income and low-income countries), though low-income countries had higher prevalence of breastfeeding at all ages. They found more differences in continued breastfeeding, with poorer people breastfeeding for longer than other groups. Differences of breastfeeding rates in different settings underline the influence of sociocultural factors. ${ }^{9}$

The importance of breastfeeding promotion is related to positive health effects of breastfeeding on children, mothers and society in general.

Among short-term outcomes in children there is a reduction in mortality ${ }^{10,11}$ and morbidity in terms of diarrhea, respiratory infections ${ }^{12}$ and otitis media, ${ }^{13}$ while there is no clear protection against allergic disorders. ${ }^{14}$ Breastfeeding is also associated with better longterm effects: increase of intelligence quotient; 15,16 reduction of overweight or obesity, type 2 diabetes ${ }^{17}$ and of childhood leukemia. ${ }^{18}$

Among mother's effects, breastfeeding is associated with prolonged amenorrhea, reduced breast and ovarian cancer. ${ }^{19}$

The aim of this study is to review the evidence-based interventions for breastfeeding support and to underline actual problems to achieve it in different settings.

\section{Evidence for breastfeeding promotion interventions}

Breastfeeding support is a complex system of interventions, varying from informational, practical, emotional and social support. Settings differ from hospital and community. People involved can be lay or health professionals, trained or not, offering proactive or reactive support, to one or more women and furnished face-to-face, by telephone or social media; contacts can be one or ongoing in months. ${ }^{20,21}$

Are therefore relevant training and education of people offering breastfeeding support, as provided by WHO and UNICEF (United Nations Children's fund): the 40-hour breastfeeding counseling and the 5-day infant and young child feeding counseling.22,23

The Baby Friendly Hospital Initiative (BFHI) includes 10 
steps to support breastfeeding and is associated to increased breastfeeding rates. ${ }^{24-26}$

Evidence of effect of breastfeeding support are of paramount importance in programming adequate interventions. In this perspective one of the most important piece of evidence comes from the PROBIT Study, a large cluster randomized controlled trial on breastfeeding promotion, with more of $80 \%$ follow-up of mother-infant pairs for 11.5 years. ${ }^{27}$ The PROBIT Study showed that breastfeeding promotion intervention modeled on BFHI increases the duration and exclusivity of breastfeeding and reduces the risk of gastrointestinal infections and atopic eczema in infancy; intervention is also associated to higher intelligence performances at 6.5 years.

Rollins proposes a conceptual model of interventions to improve breastfeeding practices based on breastfeeding determinants, divided in: individual (mother and infant characteristics and relationship), structural (sociocultural and market context) and setting factors (health services, family and community, work conditions). ${ }^{28}$ Interventions associated with increased early initiation and exclusive breastfeeding are antenatal and postnatal counseling, support and lactation management, community-based practices, better workplace conditions (availability of lactation rooms and breast milk breaks); effects are greater if interventions are provided in combination.

A recent updated Cochrane review ${ }^{29}$ confirmed the evidence that breastfeeding support offered to women increases both duration and exclusivity of breastfeeding before four to six weeks and before six months of age. Despite the great heterogeneity of the groups, the effect of supportive interventions is robust across settings.

Among the wide range of interventions, greater effect for exclusive breastfeeding is associated with face-to-face support rather than telephone support and with settings of high background breastfeeding initiation rates; also lay support and more contact with scheduled visits (4 to 8$)$ produce increased treatment effects.

Promoting breastfeeding antenatally is appealing but a recent systematic review ${ }^{30}$ fail to show any positive effect of antenatal education on initiation of breastfeeding, proportion of breastfeeding women or exclusive breastfeeding at three or six months.

On the other hand, interventions such as health education and counseling given by trained health professionals and peer support were demonstrated to have some efficacy in increasing breastfeeding starting rates, especially in ethnic and social groups with low baseline rates. ${ }^{31}$ The most effective interventions were one-to-one support and the practice of the rooming-in (allowing mothers and newborns to stay together all the time long), while surprising early mother-newborn contact was not effective in raising breastfeeding initiation. As this regard it is important to cite other systematic reviews 32 that clearly showed an effect in promoting sustained exclusive breastfeeding at four and six months and a longer duration of breastfeeding for women who were allowed to have early skin-to-skin contact with their newborns.
Breastfeeding duration is also affected by baby's father and by other relatives, with reports of longer breastfeeding in women supported by their partners. 33,34

\section{Breastfeeding promotion in Neonatal Intensive Care Unit}

If breastfeeding is of paramount importance in full term healthy newborns, it is even more important in high risk newborns, such as low and very low birth weight infants and others newborns in the NICUs. Risks associated with lack of breastfeeding are well known and include higher rates of necrotizing enterocolitis and infections, and lower scores of neurodevelopmental tests.35-39 On the other hand, rates of breastfeeding are lower in the NICU setting worldwide. 6,40 For these reasons promotion of breastfeeding is crucial in the NICUs. Many factors make breastfeeding more difficult in NICU. Maternal stress and disease, separation, delayed enteral and/oral feeding and insufficient health care skills are all critical factors related to low breastfeeding rates among high risk newborns.

Supporting breastfeeding in NICUs is part of a more wide promotion of attachment and is an essential part of a more comprehensive standard of care. Effective interventions were identified in a recent systematic review, ${ }^{41}$ including a family-centered approach to care, skin-to-skin promotion, reduction of stress and pain; other interventions are practices that support parents, promote motherinfants attachment and empower mothers in taking care of their high risk newborns.

Effective and achievable interventions that promote and support breastfeeding in NICU include: kangaroo skin-to-skin contact, simultaneous milk expression from both breasts in the early weeks after birth, peer support in hospital and community, multidisciplinary staff training, Baby Friendly accreditation of the associated maternity hospital. Feeding from a cup as opposed to a bottle increases rates of exclusive breastfeeding at discharge, but applicability of this finding may be limited.

In addition, the interventions are interrelated, with interventions less effective if implemented individually. The greatest improvements are associated with multifaceted interventions, particularly those which include staff training or provide an environment in which mothers are encouraged and supported to breastfeed or express milk while maintaining close contact with their infants. This systematic review supported the evidence of increased breastfeeding rates in neonatal units within a UNICEF Baby Friendly accredited maternity service. It is also congruent with the evidence based for term infants and their mothers, where multifaceted interventions are the most efficacious. ${ }^{42}$ Effective process of care includes support for breast milk feeding and timely initiation of breastfeeding. Kangaroo skin-to-skin contact should be encouraged as soon as infants are

Table 1. Exclusive breastfeeding rates in Italy.

\begin{tabular}{lccc} 
& & Exclusive breastfeeding (\%) & \\
& At discharge & 3 months & 6 months \\
Lauria et al. $(2016)^{3}$ & 57.2 & 48.6 & 5.5 \\
Giovannini et al. $(2003)^{4}$ & 70.0 & 36.2 & 7.6 \\
\hline Giovannini et al. $(2003)^{4}$ & 76.8 & 47.0 & 4.7 \\
Carletti et al. $(2011)^{5}$ & 69.0 & - & 6.0 \\
\hline Davanzo et al. $(2009)^{6}$ & 31.0 (VLBWI); 24.0 (LBWI) & - \\
\hline VLBWI, very low birth weight infant; LBWI, low birth weight infant. & & -
\end{tabular}


clinically stable and facilities and support provided to facilitate effective expression and storage of breast milk. Furthermore, implementation of a human donor milk bank is demonstrated to be associated with higher rate of breastfeeding, 43 underlying the importance of a culture of breastfeeding support

\section{Problems with breastfeeding promotion}

Disadvantages in terms of health and economic costs relied to low breastfeeding rates are in general underestimated. Early discontinuation of breastfeeding can be associated with maternal problems, like maternal illness, breast pain, discomfort to breastfeeding in public, or with newborn illness.

Health-care workers have a crucial role for exclusive and continued breastfeeding, because they support breastfeeding in the perinatal period and in the following days, so health professional inadequacy in solving breastfeeding problems for lack of knowledge and skills can negatively influence breastfeeding support. 44,45

Even more in a NICU contest multidisciplinary staff training is required; maximizing parent-infant contact to achieve countless beneficial effects have other implications, like improving the design of hospital facilities, giving parents' accommodation, and management of neonatal networks and transport. Surveillance of feeding for infants in NICU and following discharge is needed to inform future policy and practice development. In a research perspective, the role and the impact of public health and policy interventions, the role of family and community staff, and the organization of neonatal services deserve further investigation.

Women's work and short maternity leave are associated with not breastfeeding or early weaning. In addition, it is growing the industry marketing of breast milk substitutes that is in competition with breastfeeding support programs.

To protect and to continue promoting breastfeeding in all settings (high-income, middle-income and low-income countries) both political and economic support campaigns are necessary.

\section{Conclusions}

There is sufficient evidence to state that breastfeeding support is necessary to increase the duration and exclusivity of breastfeeding, ensuring the multiple health advantages to full term and preterm newborns, children, mothers and society in general. Efforts should be made to ensure breastfeeding support to all women in the different settings, first of all assuming it as a collective and not only a maternal goal. As breastfeeding practices are affected by different factors, interventions in promoting breastfeeding include individual, social, political and financial aspects.

\section{References}

1. WHO. Global strategy for infant and young child feeding. Geneva, Switzerland: World Health Organization; 2003.

2. International Food Policy Research Institute. Global Nutrition Report 2015: actions and accountability to advance nutrition and sustainable development. Washington, DC: International Food Policy Research Institute; 2015.

3. Lauria L, Spinelli A, Grandolfo M. Prevalence of breastfeed- ing in Italy: a population based follow-up study. Ann Ist Super Sanita 2016;52:457-61.

4. Giovannini M, Banderali G, Radaelli G, et al. Monitoring breastfeeding rates in Italy: national surveys 1995 and 1999. Acta Paediatr 2003;92:357-63.

5. Carletti C, Pani P, Knowles A, et al. Breastfeeding to 24 months of age in the northeast of Italy: a cohort study. Breastfeed Med 2011;6:177-82.

6. Davanzo R, Ronfani L, Brovedani P, et al. Breast feeding verylow-birthweight infants at discharge: a multicentre study using WHO definitions. Paediatr Perinat Epidemiol 2009;23:591-6.

7. WHO. Indicators for assessing infant and young child feeding practices. Part I: definition. Geneva, Switzerland: World Health Organization; 2008.

8. Victora C, Barros A, França G, et al. Breastfeeding in the 21st century: epidemiology, mechanisms, and lifelong effect. Lancet 2016;387:475-90.

9. EU Project on Promotion of Breastfeeding in Europe. Protection, promotion and support of breastfeeding in Europe: a blueprint for action. Luxembourg: European Commission, Directorate Public Health and Risk Assessment; 2004.

10. Sankar MJ, Sinha B, Chowdhury R, et al. Optimal breastfeeding practices and infant and child mortality. A systematic review and meta-analysis. Acta Paediatr 2015;104:3-13.

11. Ip S, Chung M, Raman G, et al. Breastfeeding and maternal and infant health outcomes in developed countries. Evid Rep Technol Assess 2007;153:1-186.

12. Horta BL, Victora CG. Short-term effects of breastfeeding: a systematic review of the benefits of breastfeeding on diarrhea and pneumonia mortality. Geneva, Switzerland: World Health Organization; 2013.

13. Bowatte G, Tham R, Allen KJ, et al. Breastfeeding and childhood acute otitis media: a systematic review and meta-analysis. Acta Paediatr Suppl 2015;104:85-95.

14. Lodge CJ, Tan DJ, Lau M, et al. Breastfeeding and asthma and allergies: a systematic review and meta-analysis. Acta Paediatr Suppl 2015;104:38-53.

15. Horta BL, de Mola CL, Victora CG. Breastfeeding and intelligence: systematic review and meta-analysis. Acta Paediatr Suppl 2015;104:14-9.

16. Victora CG, Horta BL, de Mola CL, et al. Association between breastfeeding and intelligence, educational attainment, and income at 30 years of age: a prospective birth cohort study from Brazil. Lancet Glob Health 2015;3:e199-205.

17. Horta BL, de Mola CL, Victora CG. Long-term consequences of breastfeeding on cholesterol, obesity, systolic blood pressure, and type-2 diabetes: systematic review and meta-analysis. Acta Paediatr Suppl 2015;104:30-37.

18. Amitay EL, Keinan-Boker L. Breastfeeding and childhood leukemia incidence: a meta-analysis and systematic review. JAMA Pediatr 2015;169:e151025.

19. Chowdhury R, Sinha B, Sankar MJ, et al. Breastfeeding and maternal health outcomes: a systematic review and metaanalysis. Acta Paediatr Suppl 2015;104:96-113.

20. Dykes F. The education of health practitioners supporting breastfeeding women: time for critical reflection. Mater Child Nutr 2006;2:204-16.

21. Schmied V, Beake S, Sheehan A, et al. Women's perceptions and experiences of breastfeeding support: a metasynthesis. Birth 2011;38:49-60.

22. WHO/UNICEF. Breastfeeding counselling: a training course. Geneva, Switzerland: World Health Organization; 1993. Available from: http://www.who.int/maternal_child_adolescent/documents/who_cdr_93_3/en/ 
23. WHO/UNICEF. Infant and young child feeding counselling: an integrated course. Geneva, Switzerland: World Health Organization; 2006. Available from: http:/www.who.int/maternal_child_adolescent/documents/9789241594745/en/2006

24. Venancio SI, Saldiva SR, Escuder MM, Giugliani ER. The baby-friendly hospital initiative shows positive effects on breastfeeding indicators in Brazil. J Epidemiol Commun Health 2012;66:914-8.

25. Labbok MH. Global Baby-friendly Hospital Initiative monitoring data: update and discussion. Breastf Med 2012;7:210-22.

26. Pérez-Escamilla R, Martinez JL, Segura-Pérez S. Impact of the baby-friendly hospital initiative on breastfeeding and child health outcomes: a systematic review. Mater Child Nutr 2016;12:402-17.

27. Patel R, Oken E, Bogdanovich N, et al. Cohort profile: the Promotion of Breastfeeding Intervention Trial (PROBIT). Int $\mathrm{J}$ Epidemiol 2014;43:679-90.

28. Rollins N, Bhandari N, Hajeebhoy N, et al. Why invest, and what it will take to improve breastfeeding practices? Lancet 2016;387:491-504.

29. McFadden A, Gavine A, Renfrew MJ, et al. Support for healthy breastfeeding mothers with healthly term babies (Review). Cochrane Database Syst Rev 2017;2:CD001141.

30. Lumbiganon P, Martis R, Laopaiboon M, et al. Antenatal breastfeeding education for increasing breastfeeding duration. Cochrane Database Syst Rev 2016;12:CD006425.

31. Balogun OO, O’Sullivan EJ, McFadden A, et al. Interventions for promoting the initiation of breastfeeding. Cochrane Database Syst Rev 2016;11:CD001688.

32. Moore ER, Bergman N, Anderson GC, Medley N. Early skinto-skin contact for mothers and their healthy newborn infants. Cochrane Database Syst Rev 2016;11:CD003519.

33. Bar-Yam NB, Darby L. Fathers and breastfeeding: a review of the literature. J Hum Lact 1997;13:45-50.

34. Gibson-Davis CM, Brooks-Gunn J. The association of couples' relationship status and quality with breastfeeding initia- tion. J Marriage Fam 2007;69:1107-17.

35. Narayanan I, Prakash K, Prabhakar AK, Gujral VV. A planned prospective evaluation of the anti-infective property of varying quantities of expressed breastmilk. Acta Paediatr Scand 1982;71:441-5.

36. El-Mohandes AE, Picard MB, Simmens SJ, Keiser JF. Use of human milk in the intensive care nursery decreases the incidence of nosocomial sepsis. J Perinatology 1997;17:130-4.

37. Schanler RJ. The use of human milk for premature infants. Pediatr Clin North Am 2001;48:207-19.

38. Kramer MS, Aboud F, Mironova E, et al. Breastfeeding and child cognitive development: new evidence from a large randomized trial. Arch Gen Psych 2008;65:578-84.

39. Smith, MM, Durkin, M, Hinton VJ, et al. Influence of breastfeeding on cognitive outcomes at age 6-8 years: follow-up of very low birth weight infants. Am J Epidemiol 2003;158:1075-82.

40. Furman L, Taylor G, Minich N, Hack M. The effect of maternal milk on neonatal morbidity of very low-birth-weight infants. Arch Pediatr Adoles Med 2003;157:66-71.

41. Renfrew MJ, Dyson L, McCormick F, et al. Breastfeeding promotion for infants in neonatal units: a systematic review. Child Care Health Dev 2010;36:165-78.

42. Dyson L, Renfrew MJ, Mcfadden A, et al. Promotion of breastfeeding initiation and duration. Evidence into practice briefing. London, UK: National Institute for Health and Clinical Excellence; 2006.

43. Arslanoglu S, Moro GE, Bellù R, et al. Presence of human milk bank is associated with elevated rate of exclusive breastfeeding in VLBW infants. J Perinat Med 2013;41:129-31.

44. McAllister H, Bradshaw S, Ross-Adjie G. A study of in-hospital midwifery practices that affect breastfeeding outcomes. Breastfeed Rev 2009;17:11-5.

45. Leviniene G, Petrauskiene A, Tamuleviciene E, et al. The evaluation of knowledge and activities of primary health care professionals in promoting breast-feeding. Medicina 2009;45:238-47. 